\title{
Academic and Special Library Collaboration in North Carolina: the MAHEC/WCU Partnership
}

\author{
by Timothy V. Carstens
}

\section{Introduction}

Distance education is a rapidly expanding segment of the education marketplace, and the ability to support remote students with appropriate and quality services has become a major challenge for libraries. This article is concerned with library support for nursing students who are enrolled in a distance education program. Specifically, this article describes a collaborative arrangement wherein the Mountain Area Health Education Center (MAHEC) provides Western Carolina University (WCU) students with library services. This agreement is noteworthy for three reasons. First, remote services are being provided not just by a conveniently located academic or public library (as is typically the case in distance education support) but by a specialized library appropriate to the field of study of these students. Second, because provisions have been made to provide these students with the resources of their home library, as well as the resources of other libraries in their library network, by means of an already-established document delivery service. Third, because this collaboration involves the inclusion of bibliographic records in two separate online catalogs and the simultaneous operation of two different circulation systems. These unique arrangements provide WCU nursing students with high-quality specialized on-site services while still allowing them convenient access to all of the resources that would normally be available to them at their home library at WCU. This article will describe the rationale, the history and the working details of this unique collaboration between MAHEC and WCU.

\section{Distance Education and Western Carolina University:}

While distance education is nothing new, the advent of the Internet has made it one of the fastest growing segments of the higher education marketplace. According to the National Center for Education statistics,

From 1994-95 to 1997-98, the number of course offerings and enrollments in distance education approximately doubled. And ... the number of degree and certificate programs that were offered nearly doubled.... The percentage of institutions using asynchronous Internet-based technologies, however, nearly tripled, from 22 percent of institutions in 1995 to 60 percent of institutions in $1997-98 .^{1}$

Western Carolina University has been among those universities that have aggressively developed a suite of distance education programs. WCU now provides a number of programs in a completely online format. For example, programs in criminal justice, emergency medical care, and project management are offered completely online. Hunter Library supports each of these programs with a specially-designed website that includes instructions on how to access electronic course reserves, electronic books and journals, how to order books through Ingenta or interlibrary loan, and a list of suggested websites and links to appropriate databases. WCU also provides a number of distance education programs at North Carolina community colleges through the use of interactive video technology. These programs are also supported through library web sites, databases and document delivery. Additionally, bibliographic instruction may be provided through interactive video or at the physical class site.

While WCU has been successful in its use of new technologies such as interactive video and the Internet, WCU's most well-established and prosperous distance education venture has been suite of programs offered on the campus of the University of North Carolina at Asheville 
(UNC-A), located approximately one hour from WCU. WCU professors commute to Asheville to teach these classes in person. WCU has a formal agreement with UNC-A wherein UNC-A's Ramsey Library provides library services to WCU students in Asheville. Hunter Library also selects, acquires, and catalogs library materials that are integrated into the Ramsey Library collection in support of WCU courses taught at Asheville.

One of the most successful and extensive WCU distance education ventures in Asheville is the WCU Nursing Program. The Department of Nursing was founded in 1969 and in 2003 it awarded its 1,500th bachelor/RN degree. WCU nursing graduates consistently score one of the top passing rates in North Carolina on professional licensure exams. WCU provides three separate nursing programs in Asheville: a Master of Science in nursing; a capstone program for students who already have a two-year associate degree; and a program for students completing the final year of their BSN (the earlier part of the BSN is completed at WCU's campus in Cullowhee).

\section{A new home for the Nursing program in Asheville}

Both WCU and UNC-A are growing institutions; distance education programs offered by WCU in Asheville have been growing particularly quickly. This growth has strained the physical resources available at the Asheville campus and forced WCU to look for other places to offer its Asheville classes. Fortunately, space was found at the new campus of Asheville-Buncombe Technical Community College (A-B Tech.) at Enka. With the help of a $\$ 250,800$ grant from the Kate B. Reynolds Foundation, WCU's Asheville nursing programs were relocated to the Enka campus in October 2003.

Because there are no library facilities on the Enka campus, Hunter Library was forced to look for another way to provide services to nursing students in Asheville. It soon became apparent that many nursing students were already using another library located within the Asheville area. This library, the Health Science Library of the Mountain Area Health Education Center (MAHEC), located in downtown Asheville, is adjacent to the city's major hospital, Mission St. Joseph's Hospital.

MAHEC is a member of the North Carolina AHEC program and its mission is to provide education, information, training, and services to meet the health needs of 16 western North Carolina counties. MAHEC provides major programs in family medicine, obstetrics and gynecology, and a program of regional continuing education. Research and demonstration studies on regional health-related issues are also conducted. The Health Science Library supports these activities with a staff of three librarians, a collection of 6,000 print volumes, 350 periodical subscriptions, access to 2,500 electronic journals, and numerous online databases.

MAHEC provides full Health Science Library membership and privileges to practicing health care professionals as part of its program of professional development and continuing education. Many WCU students enrolled in the masters and capstone programs are currently working as health care professionals and therefore already have MAHEC privileges. A survey designed to assess WCU nursing students' use of facilities in Asheville showed that 65\% were already using the MAHEC library and 36\% used the MAHEC library frequently. Since so many WCU nursing students were already using the MAHEC library, finding a way to make this library the official provider of library services for these students seemed like a logical step to take.

\section{A mutually beneficial agreement}

MAHEC and WCU's Hunter Library negotiated a formal agreement on the provision of services to WCU students in the fall of 2003. According to this agreement, MAHEC Health Science Library staff provides WCU nursing students with face-to-face service for circulation, reserves, reference, printing, and photocopying. In return, WCU pays the standard membership fee for every student who is not already a member. In addition, WCU agreed to house approximately 500 volumes of monographic nursing materials and ten current periodical subscriptions at MAHEC for use by both the nursing student and other MAHEC clients. WCU also agreed to add new nursing books to the MAHEC collection on an on-going basis in order to help keep the collection current. Finally, MAHEC became an official stop on the ABC Express van route. ABC Express is a document delivery service that runs between the campuses of the University of North Carolina at Asheville, Appalachian State University and Western Carolina University, all members of the Western North Carolina Library Network (WNCLN). Making MAHEC a stop on the ABC Express route allows WCU nursing students to borrow material housed at WCU or the 
other WNCLN libraries without having to travel to Cullowhee. It also enables WNCLN students to borrow the materials WCU has placed at MAHEC.

The agreement between MAHEC and WCU provides important benefits to both parties. MAHEC's most important mission is health education and the partnership with WCU provides an additional way to fulfill this mission. The agreement also expands MAHEC's collection and provides them with additional paying members. Perhaps most importantly, the agreement offers MAHEC the opportunity to work with WCU nursing students. Since many WCU students will stay in western North Carolina, these students are all potential future clients of MAHEC. Nursing students who become accustomed to using the MAHEC Health Science Library to meet their information needs as students are also likely to use MAHEC when they have information needs as practicing professionals.

Like MAHEC, Western Carolina University receives important benefits from the partnership. The staff at MAHEC is especially qualified to serve nursing students since they specialize in medical information, unlike the generalists normally found within academic libraries. Additionally, nursing students gain experience with a real medical library. Medical libraries are organized in a different way than public or academic libraries; learning to use a medical library is likely to be helpful to these students when they become practicing professionals. Finally, WCU nursing students will benefit from having access to the extensive medical collection already at MAHEC, which, when combined with the material supplied by WCU and ABC Express, provides these students with a much larger amount of information than they would have at either WCU or UNC-A. WCU nursing students can also borrow materials from the other member of the North Carolina AHEC Libraries Consortium, further increasing the amount of medical information available to them. The WCU agreement with MAHEC provides WCU nursing students with all of the advantages of having access to a specialized medical library yet still supplies them with access to the resources of larger, more general university libraries.

\section{Making it happen}

While the MAHEC/WCU collaboration is mutually beneficial for both parties, making this agreement work requires diligence. Special arrangements, training, and preparation were needed in every area of library operation. Since ABC Express services are made available through the WNCLN catalog, providing ABC Express services requires MAHEC to utilize WNCLN's catalog simultaneously with their own catalog. Furthermore, WCU material housed in MAHEC must be cataloged within both systems. This is the only way that MAHEC can both check out WCU material to their non-WCU patrons and, simultaneously, allow this material to be available for circulation via ABC Express.

Transferring WCU materials related to medicine from the UNC-A stacks (where they were previously held) to the MAHEC stacks was the first task that had to be accomplished. The WCU health sciences bibliographer, WCU Nursing faculty, and MAHEC librarians examined these materials to determine if they should be weeded from the collection, remain in Asheville, or be transferred to MAHEC. Duplication with the MAHEC collection was also checked at this point. Once identified, this material was pulled from the UNC-A stacks and sent to the technical services units at WCU.

At WCU, this material was processed in several ways. First, these materials had to be reclassified from the Library of Congress classification used by WNCLN to the NLM classification system used by MAHEC. Since neither MAHEC nor WCU had catalogers trained in the NLM classification system, this reclassification was accomplished by accepting the NLM call numbers found in OCLC records for this material. Call numbers were found in OCLC for most of this material; the cataloger at Coastal AHEC (which shares MAHEC's online catalog) agreed to provide call numbers of the few remaining items. Records for the materials in the WNCLN catalog were then changed to reflect the new call number and location. In addition, 949 fields were added to these records at this time. These 949 fields create item records in the MAHEC catalog that include call number, location and circulation status information. The records were then exported from WCU's Innopac system, sent via File Transfer Protocol (FTP) to MAHEC and loaded into the MAHEC catalog. The last step in the process was to relabel and reprocess the material for use at MAHEC and to send it on to that library. At the end of these processes, all WCU materials at MAHEC could be searched for and circulated in both the WCU and the MAHEC catalog.

Another important part of establishing the WCU/MAHEC collaboration was the configuration of the computer systems of the two orga- 
nizations. Since the two organizations have different circulation loan periods, rules for renewal, fines, etc. they had to agree on what rules would apply to WCU students and regular MAHEC patrons who were using the new joint collection. These rules were then entered into the administrative module of the online systems. Firewalls presented a more complex computer-related problem. Because patient confidentiality is critical for them, health service organizations tend to establish especially impenetrable firewalls. MAHEC's firewall initially interfered with necessary communication with the WCU system. Due to the firewall, access to WCU's online catalog was available to MAHEC staff via Telnet but was not available via the web. Because of this problem, WCU students at MAHEC were unable to search for materials located on the WCU campus which should have been available to them via ABC Express. Furthermore, MAHEC's computer would not accept new catalog records created by WCU and sent to them via FTP and there was difficulty printing of item pull slips for the ABC Express system. Each of these firewall problems was resolved after negotiation and consultation between the systems librarian at WCU and the computer networking staff at MAHEC.

Another issue that the two libraries had to resolve concerned handling money. The amount of money collected in fines for overdue or damaged materials and the procedures for collecting those fines were negotiated and determined. Another problem to be resolved was the difference in printing costs between WCU and MAHEC. MAHEC charges more than WCU for both computer printing and photocopying. To resolve this problem, procedures were established whereby WCU students would pay for these charges at the WCU rate with Hunter Library reimbursing MAHEC for the difference between this rate and the MAHEC rate.

Once records were in the catalog, computer systems established and working, and policies established, MAHEC staff had to be trained to use the WNCLN circulation system. This was done during an intensive half-day session that concentrated on the ABC Express aspects of the III circulation system. A training manual was also created by WCU for the use of MAHEC staff.

\section{Conclusion}

The MAHEC Health Science Library has been providing library services to WCU nursing students since September 2003. The arrangement has been working smoothly. WCU students are receiving reference service at MAHEC, circulation and ABC Express services have been working as planned, and additional materials purchased and cataloged by WCU have been added to the MAHEC stacks on a regular basis. These arrangements are unique in two ways. First, distance education students receive library support from a library that specializes in their field of study. Second, this arrangement involves the expansion of an existing document delivery service and the integration of a collection of library resources into two different integrated library systems.

As the MAHEC/WCU relationship matures, other areas for further cooperation will be explored. The WCU health science liaison will need to work closely with MAHEC and the WCU nursing faculty in Asheville to ensure that the best possible bibliographic instruction is provided to WCU nursing students. The two libraries also need to develop processes for cooperative collection development. WCU materials purchased for MAHEC should not only support WCU nursing students, these materials must also enhance the MAHEC collection as a whole. In addition, these materials must also complement those materials available through ABC Express and through AHEC LARC consortium. The MAHEC Health Science Library and WCU's Hunter Library are sure to explore these areas and others as they continue to enjoy their successful and mutually beneficial alliance.

\section{References:}

${ }^{1}$ National Center for Education Statistics, "Distance Education at Postsecondary Education Institutions: 1997-98, Executive Summary,” <http://nces.ed.gov/surveys/peqis/publications/2000013/> (date accessed: November 30, 2004) 